\title{
POLYNOMIALS WITH POLAR DERIVATIVES
}

\author{
AbDullah Mir, AJaz Wani
}

\begin{abstract}
In this paper, we present an integral inequality for the polar derivative of polynomials. Our theorem includes as special cases several interesting generalizations of some Zygmund type inequalities for polynomials.
\end{abstract}

Keywords: polar derivative, Zygmund inequality.

\section{Introduction}

Let $P_{n}$ be the class of polynomials of degree $n$ and we define $D_{\alpha} P(z)=n P(z)+$ $(\alpha-z) P^{\prime}(z)$, the polar derivative of the polynomial $P(z)$ with respect to the point $\alpha$. The polynomial $D_{\alpha} P(z)$ is of degree at most $n-1$ and it generalizes the ordinary derivative in the sense that

$$
\lim _{\alpha \rightarrow \infty} \frac{D_{\alpha} P(z)}{\alpha}=P^{\prime}(z)
$$

Various results have been obtained for polar derivatives of polynomials. Aziz and Rather [1] proved that, if $P \in P_{n}$ and $P(z) \neq 0$ in $|z|<1$, then for every complex number $\alpha$ with $|\alpha| \geqslant 1$ and $r \geqslant 1$,

$$
\left\{\int_{0}^{2 \pi}\left|D_{\alpha} P\left(e^{i \theta}\right)\right|^{r} d \theta\right\}^{\frac{1}{r}} \leqslant n(|\alpha|+1) C_{r}\left\{\int_{0}^{2 \pi}\left|P\left(e^{i \theta}\right)\right|^{r} d \theta\right\}^{\frac{1}{r}},
$$

where

$$
C_{r}=\left\{\frac{1}{2 \pi} \int_{0}^{2 \pi}\left|1+e^{i \theta}\right|^{r} d \theta\right\}^{\frac{-1}{r}} .
$$

The work is sponsored by UGC, Govt. of India under the Major Research Project Scheme vide no. MRP-MAJOR-MATH-2013-29143.

2010 Mathematics Subject Classification: primary: 30A10; secondary: 30C10, 30C15 


\section{Main results}

In this paper, we generalize the above result for $r>0$. The above inequality will be a consequence from the more fundamental inequality presented by the following theorem:

Theorem. If $P \in P_{n}$ and $P(z) \neq 0$ in $|z|<1$, then for every complex numbers $\alpha, \beta$ with $|\alpha| \geqslant 1,|\beta| \leqslant 1$ and $r>0$,

$$
\begin{aligned}
& \left\{\int_{0}^{2 \pi}\left|e^{i \theta} D_{\alpha} P\left(e^{i \theta}\right)+n \beta\left(\frac{|\alpha|-1}{2}\right) P\left(e^{i \theta}\right)\right|^{r} d \theta\right\}^{\frac{1}{r}} \\
& \leqslant n\{(|\alpha|+1)+|\beta|(|\alpha|-1)\} C_{r}\left\{\int_{0}^{2 \pi}\left|P\left(e^{i \theta}\right)\right|^{r} d \theta\right\}^{\frac{1}{r}},
\end{aligned}
$$

where $C_{r}$ is defined by (1.2).

Remark. If we put $\beta=0$, in the above result, we get (1.1) extended to the case $r \in(0, \infty)$. Dividing the two sides of (2.1) by $|\alpha|$ and letting $|\alpha| \rightarrow \infty$, we get the following result.

Corollary. If $P \in P_{n}$ and $P(z) \neq 0$ in $|z|<1$, then for every complex number $\beta$ with $|\beta| \leqslant 1$ and $r>0$,

$$
\left\{\int_{0}^{2 \pi}\left|e^{i \theta} P^{\prime}\left(e^{i \theta}\right)+\frac{n \beta}{2} P\left(e^{i \theta}\right)\right|^{r} d \theta\right\}^{\frac{1}{r}} \leqslant n(|\beta|+1) C_{r}\left\{\int_{0}^{2 \pi}\left|P\left(e^{i \theta}\right)\right|^{r} d \theta\right\}^{\frac{1}{r}}
$$

where $C_{r}$ is defined by (1.2).

The above inequality generalizes some inequalities obtained by De-Bruijn [3] as well as Rahman and Schmeisser [5].

\section{Lemmas}

For the proof of the Theorem, we need the following lemmas:

Lemma 1. If $Q \in P_{n}$ be a polynomial such that $Q(z) \neq 0$ for $|z|>1$ and $P \in P_{n}$. If $|P(z)| \leqslant|Q(z)|$ for $|z|=1$, then for all complex numbers $\alpha, \beta$ with $|\alpha| \geqslant 1,|\beta| \leqslant 1$,

$$
\left|z D_{\alpha} P(z)+n \beta\left(\frac{|\alpha|-1}{2}\right) P(z)\right| \leqslant\left|z D_{\alpha} Q(z)+n \beta\left(\frac{|\alpha|-1}{2}\right) Q(z)\right|, \quad \text { for }|z| \geqslant 1 .
$$

The above lemma is due to Liman, Mohapatra and Shah [4].

Lemma 2. If $P \in P_{n}$ and $Q(z)=z^{n} \overline{P(1 / \bar{z})}$, then for every $r>0$ and $\gamma$ real,

$$
\int_{0}^{2 \pi} \int_{0}^{2 \pi}\left|P^{\prime}\left(e^{i \theta}\right)+e^{i \gamma} Q^{\prime}\left(e^{i \theta}\right)\right|^{r} d \theta d \gamma \leqslant 2 \pi n^{r} \int_{0}^{2 \pi}\left|P\left(e^{i \theta}\right)\right|^{r} d \theta .
$$

The above lemma is due to Aziz and Rather [2]. 


\section{Proof of the Theorem}

Since $P \in P_{n}$ and $P(z) \neq 0$ in $|z|<1$, the polynomial $Q(z)=z^{n} \overline{P(1 / \bar{z})} \in P_{n}$ and $Q(z) \neq 0$ in $|z|>1$. By Lemma 1 , we have for complex numbers $\alpha, \beta$ with $|\alpha| \geqslant 1,|\beta| \leqslant 1$,

$$
\left|z D_{\alpha} P(z)+n \beta\left(\frac{|\alpha|-1}{2}\right) P(z)\right| \leqslant\left|z D_{\alpha} Q(z)+n \beta\left(\frac{|\alpha|-1}{2}\right) Q(z)\right|, \quad \text { for }|z|=1 \text {. }
$$

Now for every real $\gamma$ and $t \geqslant 1$, it can be easily verified that $\left|t+e^{i \gamma}\right| \geqslant\left|1+e^{i \gamma}\right|$.

Observe that for any $r>0$ and $a, b \in \mathbb{C}$ such that $|b| \geqslant|a|$ we have

$$
\int_{0}^{2 \pi}\left|a+e^{i \gamma} b\right|^{r} d \gamma \geqslant|a|^{r} \int_{0}^{2 \pi}\left|1+e^{i \gamma}\right|^{r} d \gamma
$$

Indeed, if $a=0$ the above inequality (4.2) is obvious. In the case of $a \neq 0$ we get

$$
\begin{aligned}
\int_{0}^{2 \pi}\left|1+e^{i \gamma} \frac{b}{a}\right|^{r} d \gamma & =\int_{0}^{2 \pi}\left|1+e^{i \gamma}\right| \frac{b}{a}||^{r} d \gamma=\int_{0}^{2 \pi}|| \frac{b}{a}\left|+e^{i \gamma}\right|^{r} d \gamma \\
& \geqslant \int_{0}^{2 \pi}\left|1+e^{i \gamma}\right|^{r} d \gamma .
\end{aligned}
$$

Now, we can take

$$
a=e^{i \theta} D_{\alpha} P\left(e^{i \theta}\right)+n \beta\left(\frac{|\alpha|-1}{2}\right) P\left(e^{i \theta}\right), b=e^{i \theta} D_{\alpha} Q\left(e^{i \theta}\right)+n \beta\left(\frac{|\alpha|-1}{2}\right) Q\left(e^{i \theta}\right),
$$

because $|b| \geqslant|a|$ from (4.1), we get from (4.2) that

$$
\begin{aligned}
\int_{0}^{2 \pi} \mid\left\{e^{i \theta} D_{\alpha}\right. & \left.P\left(e^{i \theta}\right)+n \beta\left(\frac{|\alpha|-1}{2}\right) P\left(e^{i \theta}\right)\right\} \\
& +\left.e^{i \gamma}\left\{e^{i \theta} D_{\alpha} Q\left(e^{i \theta}\right)+n \beta\left(\frac{|\alpha|-1}{2}\right) Q\left(e^{i \theta}\right)\right\}\right|^{r} d \gamma \\
\geqslant & \left|e^{i \theta} D_{\alpha} P\left(e^{i \theta}\right)+n \beta\left(\frac{|\alpha|-1}{2}\right) P\left(e^{i \theta}\right)\right|^{r} \int_{0}^{2 \pi}\left|1+e^{i \gamma}\right|^{r} d \gamma .
\end{aligned}
$$

Integrating both sides of (4.3) with respect to $\theta$ from 0 to $2 \pi$, we get

$$
\begin{aligned}
\int_{0}^{2 \pi} \int_{0}^{2 \pi} \mid\left\{e^{i \theta} D_{\alpha} P\left(e^{i \theta}\right)+n \beta\left(\frac{|\alpha|-1}{2}\right) P\left(e^{i \theta}\right)\right\} \\
\quad+\left.e^{i \gamma}\left\{e^{i \theta} D_{\alpha} Q\left(e^{i \theta}\right)+n \beta\left(\frac{|\alpha|-1}{2}\right) Q\left(e^{i \theta}\right)\right\}\right|^{r} d \theta d \gamma \\
\geqslant \int_{0}^{2 \pi}\left|e^{i \theta} D_{\alpha} P\left(e^{i \theta}\right)+n \beta\left(\frac{|\alpha|-1}{2}\right) P\left(e^{i \theta}\right)\right|^{r} d \theta \int_{0}^{2 \pi}\left|1+e^{i \gamma}\right|^{r} d \gamma .
\end{aligned}
$$


As $Q(z)=z^{n} \overline{P(1 / \bar{z})}$, we have $P(z)=z^{n} \overline{Q(1 / \bar{z})}$. It can be easily verified that for $0 \leqslant \theta<2 \pi$,

$$
n P\left(e^{i \theta}\right)-e^{i \theta} P^{\prime}\left(e^{i \theta}\right)=e^{i(n-1) \theta} \overline{Q^{\prime}\left(e^{i \theta}\right)}
$$

and

$$
n Q\left(e^{i \theta}\right)-e^{i \theta} Q^{\prime}\left(e^{i \theta}\right)=e^{i(n-1) \theta} \overline{P^{\prime}\left(e^{i \theta}\right)}
$$

Hence

$$
\begin{aligned}
n P\left(e^{i \theta}\right)+e^{i \gamma} n Q\left(e^{i \theta}\right)= & e^{i \theta} P^{\prime}\left(e^{i \theta}\right)+e^{i(n-1) \theta} \overline{Q^{\prime}\left(e^{i \theta}\right)} \\
& +e^{i \gamma}\left(e^{i \theta} Q^{\prime}\left(e^{i \theta}\right)+e^{i(n-1) \theta} \overline{P^{\prime}\left(e^{i \theta}\right)}\right) \\
= & e^{i \theta}\left(P^{\prime}\left(e^{i \theta}\right)+e^{i \gamma} Q^{\prime}\left(e^{i \theta}\right)\right) \\
& +e^{i(n-1) \theta}\left(\overline{Q^{\prime}\left(e^{i \theta}\right)}+e^{i \gamma} \overline{P^{\prime}\left(e^{i \theta}\right)}\right),
\end{aligned}
$$

which gives

$$
\begin{aligned}
n\left|P\left(e^{i \theta}\right)+e^{i \gamma} Q\left(e^{i \theta}\right)\right| & \leqslant\left|P^{\prime}\left(e^{i \theta}\right)+e^{i \gamma} Q^{\prime}\left(e^{i \theta}\right)\right|+\left|\overline{Q^{\prime}\left(e^{i \theta}\right)}+e^{i \gamma} \overline{P^{\prime}\left(e^{i \theta}\right)}\right| \\
& =2\left|P^{\prime}\left(e^{i \theta}\right)+e^{i \gamma} Q^{\prime}\left(e^{i \theta}\right)\right| .
\end{aligned}
$$

Also, we have

$$
\begin{aligned}
& \left|D_{\alpha} P\left(e^{i \theta}\right)+e^{i \gamma} D_{\alpha} Q\left(e^{i \theta}\right)\right| \\
& =\left|n P\left(e^{i \theta}\right)+\left(\alpha-e^{i \theta}\right) P^{\prime}\left(e^{i \theta}\right)+e^{i \gamma}\left(n Q\left(e^{i \theta}\right)+\left(\alpha-e^{i \theta}\right) Q^{\prime}\left(e^{i \theta}\right)\right)\right| \\
& =\mid\left(n P\left(e^{i \theta}\right)-e^{i \theta} P^{\prime}\left(e^{i \theta}\right)\right)+e^{i \gamma}\left(n Q\left(e^{i \theta}\right)-e^{i \theta} Q^{\prime}\left(e^{i \theta}\right)\right) \\
& +\alpha\left(P^{\prime}\left(e^{i \theta}\right)+e^{i \gamma} Q^{\prime}\left(e^{i \theta}\right)\right) \\
& =\left|\left(\overline{Q^{\prime}\left(e^{i \theta}\right)}+e^{i \gamma} \overline{P^{\prime}\left(e^{i \theta}\right)}\right) e^{i(n-1) \theta}+\alpha\left(P^{\prime}\left(e^{i \theta}\right)+e^{i \gamma} Q^{\prime}\left(e^{i \theta}\right)\right)\right| \\
& \leqslant \overline{\left|P^{\prime}\left(e^{i \theta}\right)+e^{i \gamma} Q^{\prime}\left(e^{i \theta}\right)\right|}+|\alpha|\left|P^{\prime}\left(e^{i \theta}\right)+e^{i \gamma} Q^{\prime}\left(e^{i \theta}\right)\right| \\
& =(|\alpha|+1)\left|P^{\prime}\left(e^{i \theta}\right)+e^{i \gamma} Q^{\prime}\left(e^{i \theta}\right)\right| .
\end{aligned}
$$


Using (4.5) and (4.6), we have for $0 \leqslant \theta<2 \pi,|\alpha| \geqslant 1,|\beta| \leqslant 1$, and $\gamma$ real,

$$
\begin{aligned}
\mid\left\{e^{i \theta} D_{\alpha} P\right. & \left.\left(e^{i \theta}\right)+n \beta\left(\frac{|\alpha|-1}{2}\right) P\left(e^{i \theta}\right)\right\} \\
& +e^{i \gamma}\left\{e^{i \theta} D_{\alpha} Q\left(e^{i \theta}\right)+n \beta\left(\frac{|\alpha|-1}{2}\right) Q\left(e^{i \theta}\right)\right\} \mid \\
\leqslant & \left|D_{\alpha} P\left(e^{i \theta}\right)+e^{i \gamma} D_{\alpha} Q\left(e^{i \theta}\right)\right|+n|\beta|\left(\frac{|\alpha|-1}{2}\right)\left|P\left(e^{i \theta}\right)+e^{i \gamma} Q\left(e^{i \theta}\right)\right| \\
\leqslant & (|\alpha|+1)\left|P^{\prime}\left(e^{i \theta}\right)+e^{i \gamma} Q^{\prime}\left(e^{i \theta}\right)\right|+|\beta|\left(\frac{|\alpha|-1}{2}\right) 2\left|P^{\prime}\left(e^{i \theta}\right)+e^{i \gamma} Q^{\prime}\left(e^{i \theta}\right)\right| \\
& =\{(|\alpha|+1)+|\beta|(|\alpha|-1)\}\left|P^{\prime}\left(e^{i \theta}\right)+e^{i \gamma} Q^{\prime}\left(e^{i \theta}\right)\right| .
\end{aligned}
$$

This gives with the help of Lemma 2 for each $r>0$ and $\gamma$ real,

$$
\begin{aligned}
& \int_{0}^{2 \pi} \int_{0}^{2 \pi} \mid\left\{e^{i \theta} D_{\alpha} P\left(e^{i \theta}\right)+n \beta\left(\frac{|\alpha|-1}{2}\right) P\left(e^{i \theta}\right)\right\} \\
&+\left.e^{i \gamma}\left\{e^{i \theta} D_{\alpha} Q\left(e^{i \theta}\right)+n \beta\left(\frac{|\alpha|-1}{2}\right) Q\left(e^{i \theta}\right)\right\}\right|^{r} d \theta d \gamma \\
& \leqslant\{(|\alpha|+1)+|\beta|(|\alpha|-1)\} \int_{0}^{2 \pi} \int_{0}^{2 \pi}\left|P^{\prime}\left(e^{i \theta}\right)+e^{i \gamma} Q^{\prime}\left(e^{i \theta}\right)\right|^{r} d \theta d \gamma . \\
& \leqslant 2 \pi n^{r}\{(|\alpha|+1)+|\beta|(|\alpha|-1)\}_{0}^{r}\left|P\left(e^{i \theta}\right)\right|^{r} d \theta .
\end{aligned}
$$

From (4.4) we have for every $\alpha, \beta$ with $|\alpha| \geqslant 1,|\beta| \leqslant 1$, and $r>0$,

$$
\begin{aligned}
\int_{0}^{2 \pi} \mid e^{i \theta} D_{\alpha} P\left(e^{i \theta}\right)+n \beta & \left.\left(\frac{|\alpha|-1}{2}\right) P\left(e^{i \theta}\right)\right|^{r} d \theta \int_{0}^{2 \pi}\left|1+e^{i \gamma}\right|^{r} d \gamma \\
& \leqslant 2 \pi n^{r}\{(|\alpha|+1)+|\beta|(|\alpha|-1)\}^{r} \int_{0}^{2 \pi}\left|P\left(e^{i \theta}\right)\right|^{r} d \theta
\end{aligned}
$$

which is equivalent to $(2.1)$.

Acknowledgements. The authors are very grateful to the referee for his comments and valuable suggestions regarding the paper.

\section{References}

[1] A. Aziz and N. A. Rather, On an inequality concerning the polar derivative of a polynomial, Proc. Indian Acad. Sci. (Math. Sci.) 117 (2003), 349-357. 
[2] A. Aziz and N. A. Rather, Some Zygmund type $L^{q}$ inequalities for polynomials, J. Math. Anal. Appl. 289 (2004), 14-29.

[3] N. G. De-Bruijn, Inequalities concerning polynomials in the complex domain, Nedral. Akad., Wetnesch. Proc. 50 (1947), 1265-1272, Indag. Math. 9 (1947), 591-598.

[4] A. Liman, R. N. Mohapatra and W. M. Shah, Inequalities for the polar derivative of a polynomial, Complex Anal. Oper. Theory 6 (2012), 1199-1209.

[5] Q. I. Rahman and G. Schmeisser, $L^{p}$ inequalities for polynomials, J. Approx. Theory 53 (1988), 26-32.

Address: Abdullah Mir and Ajaz Wani: Department of Mathematics, University of Kashmir, Srinagar, 190006, India.

E-mail: mabdullah_mir@yahoo.co.in, theajazwani@yahoo.com

Received: 4 September 2015; revised: 29 June 2016 